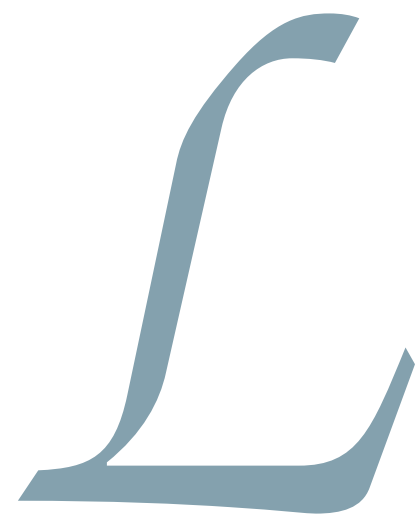

\title{
a documentación del
} proyecto y el

proyecto como documento

\section{EL PROYECTO COMO DOCUMENTO DEL MONUMENTO}

Viollet le Duc ya planteaba, como parte esencial de su metodología de restauración en estilo, la necesidad de una investigación previa a toda intervención, y puntualizaba que ésta debía de ser exhaustiva, documental e histórica, con objeto de profundizar en el complejo conocimiento del monumento. Establecía también que el estudio físico-constructivo del edificio, basado en el análisis racional de su estructura era una premisa indispensable a la hora de abordar cualquier actuación. Esta investigación documental e histórica, la completaba con levantamientos gráficos y fotográficos, considerando a todos ellos medios imprescindibles para elaborar una metodología de trabajo coherente $(\mathrm{I})$.

Pero no es hasta que, una vez superada la valoración del edificio desde un punto puramente estilístico defendida por Viollet le Duc y el mero reconocimiento de su valor romántico e histórico preconizada por Ruskin, que el teórico Camilo Boito consagre el concepto restauro moderno reconociendo al monumento como una obra dual, arquitectónica e histórica a la vez. En consecuencia, a partir de entonces todas las aportaciones y añadidos realizados en distintas épocas adquieren la misma valoración, por lo que ya no se pueden eliminar en búsqueda de aquel aspecto primigenio. Este autor siguiendo la historiografía positivista de actualidad a finales del siglo XIX profundiza, mediante la aplicación del método filológico, en el análisis del documento que supone la obra arquitectónica. Además de esta investigación previa a la actuación advierte, a los profesionales que se dedican a este tema, acerca de la necesidad de documentar la restauración con fotografías, dibujos y levantamientos planimétricos.

En España, en los años treinta, los restauradores más comprometidos y a avanzados del momento asumen la metodología de Camillo Boito, sistematizada más tarde por Giovannoni, que era útil y aplicable genéricamente a todos los edificios; entendiéndose siem- pre que el valor artístico del monumento estaba subordinado a las aportaciones que había sufrido cada arquitectura, a su evolución constructiva. Se potencia con ello el interés documentalista e histórico a costa de los valores tipológicos justificando así, la mayor parte de las veces, la presencia de añadidos y elementos extraños al edificio. La documentación del monumento era fundamental en las actuaciones en el patrimonio arquitectónico.

La Carta de Atenas (193I), en su apartado VI, reconoce al monumento como documento de civilización y cultura; a pesar de ello, no especifica ningún tipo de recomendación con relación a la documentación previa a la intervención y únicamente especifica que las supresiones de la fábrica deben quedar correctamente documentadas y expuestas en un lugar próximo del monumento.

La Carta del Restauro, también de 1931, le dedica una especial atención al tema:

"Como en las excavaciones, así también en la restauración de los monumentos será condición esencial y determinante que una documentación precisa acompañe a los trabajos además de una relación analítica recogida en un diario de restauración e ilustrada con dibujos y fotografias, de modo que todos los elementos determinados en la estructura y en la forma del monumento, todas las fases de la obra de recomposición, de liberación, de complementación, queden reflejadas de modo permanente y seguro" (2).

El historiador de arte Cesare Brandi, recogiendo toda la tradición anterior, plantea que es ineludible la historicidad de cualquier objeto arquitectónico por el hecho de ser un artefacto producido por la actividad humana, puesto que lo histórico no se circunscribe al momento concreto de la conformación original, sino que incluye toda manifestación posterior, toda huella del transcurso del tiempo incluso la última restauración, entendida ésta como una nueva aportación, un cambio o un dato más aportado a la fábrica, es decir, como una obra de arte en sí misma (3). En su teoría

\section{Concha Fontela San Juan}

Profesora del

Departamento de Historia del Arte

Universidad de Santiago 


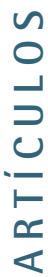

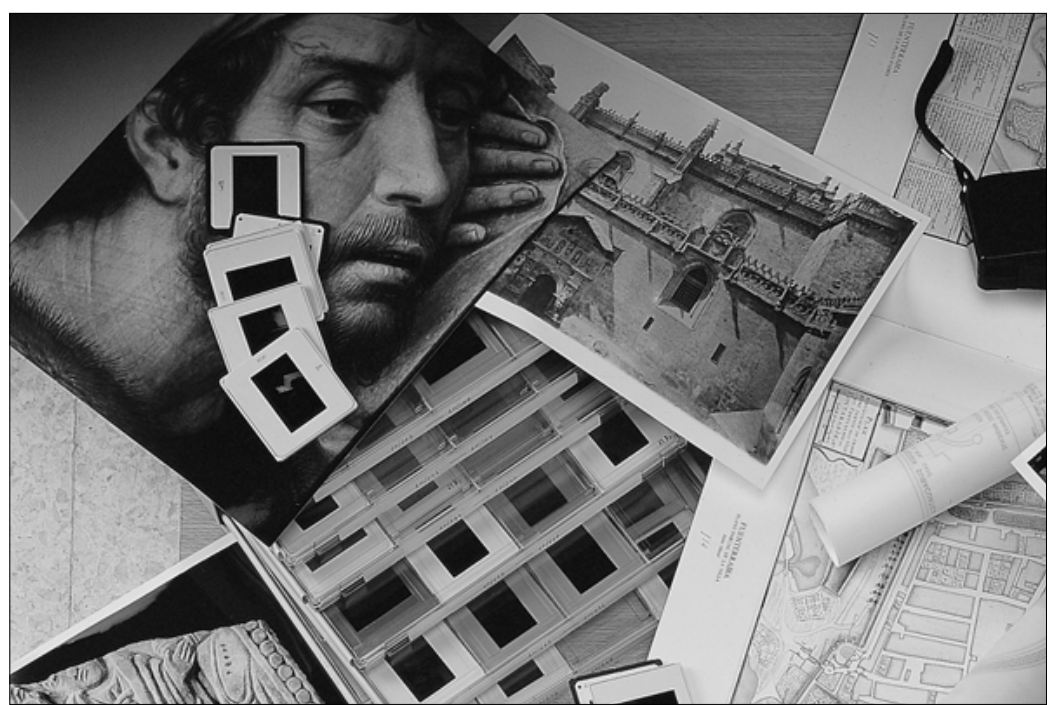

En cuanto a las recomendaciones internacionales del momento, se produce un importante avance con la Carta de Venecia de 1964 que, ya en su preámbulo, establece las bases de su desarrollo posterior al considerar que el patrimonio histórico es portador de un mensaje cultural del pasado y que las obras monumentales de cada pueblo son el testimonio vivo de sus tradiciones seculares. Por ello, la humanidad, que cada día toma más conciencia de la unidad de los valores humanos, las considera como patrimonio común y se reconoce solidariamente responsable de su conservación para su transmisión a generaciones futuras (4).

Se admite, por primera vez, en un documento de ámbito internacional que "La conservación y restauración de los monumentos constituye una disciplina que reclama la colaboración con todas las ciencias y con todas las técnicas que pueden contribuir al estudio y a la protección del patrimonio monumental" (5); y especifica en el artículo nueve:

“La restauración estará siempre precedida y acompañada por un estudio arqueológico e histórico del monumento" (6).

En la Carta del Restauro de 1972, inspirada en la teoría de Cesare Brandi, de nuevo se reclama que cada intervención debe ser estudiada previamente y argumentada por escrito. Durante el desarrollo de las obras se aconseja la realización de un diario de las operaciones efectuadas, al que seguirán la redacción final con la documentación gráfica de antes, durante y después de la intervención. Se intensifica, por tanto, no sólo la necesidad del estudio previo a la actuación sino la documentación del seguimiento de las obras y el informe final de los resultados obtenidos (7).

En la Declaración de Amsterdam de 1975 se vuelve a insistir en la necesidad de la colaboración interdisciplinar para las actuaciones en el patrimonio cultural, no sólo en los estudios históricos previos a las actuaciones, el análisis de los materiales y las técnicas constructivas utilizadas a lo largo del tiempo, sino también en la elaboración de catálogos o en la divulgación del patrimonio arquitectónico como medio indispensable para concienciar a los jóvenes de la importancia de su conservación (8).

La Ley 16/1985, de 25 de junio, del Patrimonio Histórico Español, no plantea importantes innovaciones al respecto. Sin embargo, en el apartado 3 del artículo treinta y nueve, España se adscribe a las corrientes iniciadas en Italia por Camilo Boito, y lo hace en los siguientes términos:

"Las restauraciones de los bienes a que se refiere el presente artículo respetarán las aportaciones de todas las épocas existentes. La eliminación de alguna de ellas sólo se autorizará con carácter excepcional y siempre que los elementos que traten de suprimirse supongan una evidente degradación del bien y su eliminación fuere necesaria para permitir una mejor interpretación histórica del mismo. Las partes suprimidas quedarán debidamente documentadas" (9).

A pesar del gran número de alusiones al que se ha hecho referencia, al enorme interés despertado por los valores documentales, históricos y estéticos de los monumentos, y aunque tanto en las primeras recomendaciones internacionales y cartas del restauro, como en la legislación nacional sobre su conservación se hace referencia expresa a las excavaciones arqueológicas y a la necesidad de colaboración entre arqueólogos y arquitectos, el papel de los historiadores está poco determinado y, generalmente, al margen en dichas recomendaciones internacionales y nacionales en materia de patrimonio cultural.

\section{LA DOCUMENTACIÓN HISTÓRICA EN LOS PROYECTOS DE RESTAURACIÓN REALIZADOS EN GALICIA ENTRE 1980 Y 1990}

En base a los datos recopilados en la investigación realizada, en la década de los años ochenta, se ha podido constatar como la mayor parte de las memorias de los proyectos de restauración arquitectónica no recogen ni tan siquiera los datos mínimos necesarios para una primera comprensión del edificio. Los estudios previos a las actuaciones resultan insustituibles si el arquitecto pretende lograr una correcta interpretación de la fábrica. Sólo profundizado en la de evolución constructiva de la fábrica podrá llegar a conocer su correcta situación actual y deducir la diagnosis que solucione las patologías que presente. Así, mediante la utilización de las soluciones cons- 
tructivas más adecuadas y aplicando los criterios de restauración con el rigor científico que requiere una actuación en un Bien de Interés Cultural, puede redactar primero y dirigir después un adecuado proyecto de restauración.

Es necesario considerar al respecto que un objeto arquitectónico es, esencialmente, un espacio útil al hombre, definido por unos elementos físicos que, como el espacio mismo, adquieren una forma condicionada por la función (entendida como utilidad y como significación) y por la necesidad de sustentación, que se expresa plásticamente mediante los postulados culturales de su época. "La doble consideración del monumento como documento histórico y como objeto arquitectónico da pie a una colaboración interdisciplinar basada en el respeto mutuo" (10).

El arquitecto catalán Antoní González mantiene que, en la mayoría de los casos, los objetivos tendrán que ser definidos desde una valoración arquitectónica pero que, en todos los casos, su tratamiento científco como documento sugerirá objetivos secundarios e influirá en la metodología que se habrá de utilizar, incluso en la más específicamente proyectual.

En la actualidad, este aspecto de la restauración arquitectónica es frecuentemente olvidado por los arquitectos ya que la mayor parte consideran que la memoria histórica es un trámite más a cubrir en la presentación oficial de un proyecto de restauración. Puede buscarse, como pretexto, que desconocen la rentabilidad proyectual que les puede proporcionar una adecuada lectura científica del monumento. Sin embargo, en los pocos casos en los que esta cooperación ha sido posible los resultados han sido interesantes y fructíferos para ambos profesionales.

Admitir que el monumento es un documento supone que cualquier intervención realizada en un edificio, considerando portador de una rica información estratificada en sus fábricas, ha de afrontarse con el mayor grado de conocimiento previo y diferencia posibles. Para ello es necesario plantearse la revisión de todas las disciplinas que, de un modo u otro, intervienen en el proceso de restauración monumental. Desde los arqueólogos, historiadores, historiadores de arte, químicos, restauradores a los arquitectos, todos necesitan una profunda reflexión para readaptar su metodología individual de trabajo, a una colaboración interdisciplinar.

El arqueólogo Agustín Azcárate, recuerda la tantas veces repetida y vieja metáfora que compara el trabajo de un arqueólogo con el conocimiento de un libro cuyas páginas el lector quema según avanza en su lectura:

"Aunque no parece que los arqueólogos hayamos aprendido demasiado del ejemplo, a juzgar por la insistencia y alegría con las que se excavan yacimientos de todo tipo, también es cierto que existen todavía arquitectos no conscientes del carácter inevitablemente destructivo de su trabajo y proyectan restauraciones en edificios de indudable valor histórico pensando que un pequeño capí- tulo encargado a un joven licenciado en Historia -casi inevitablemente en paro- es suficiente para cumplir con sus obligaciones respecto al carácter monumental del edificio" ( I I).

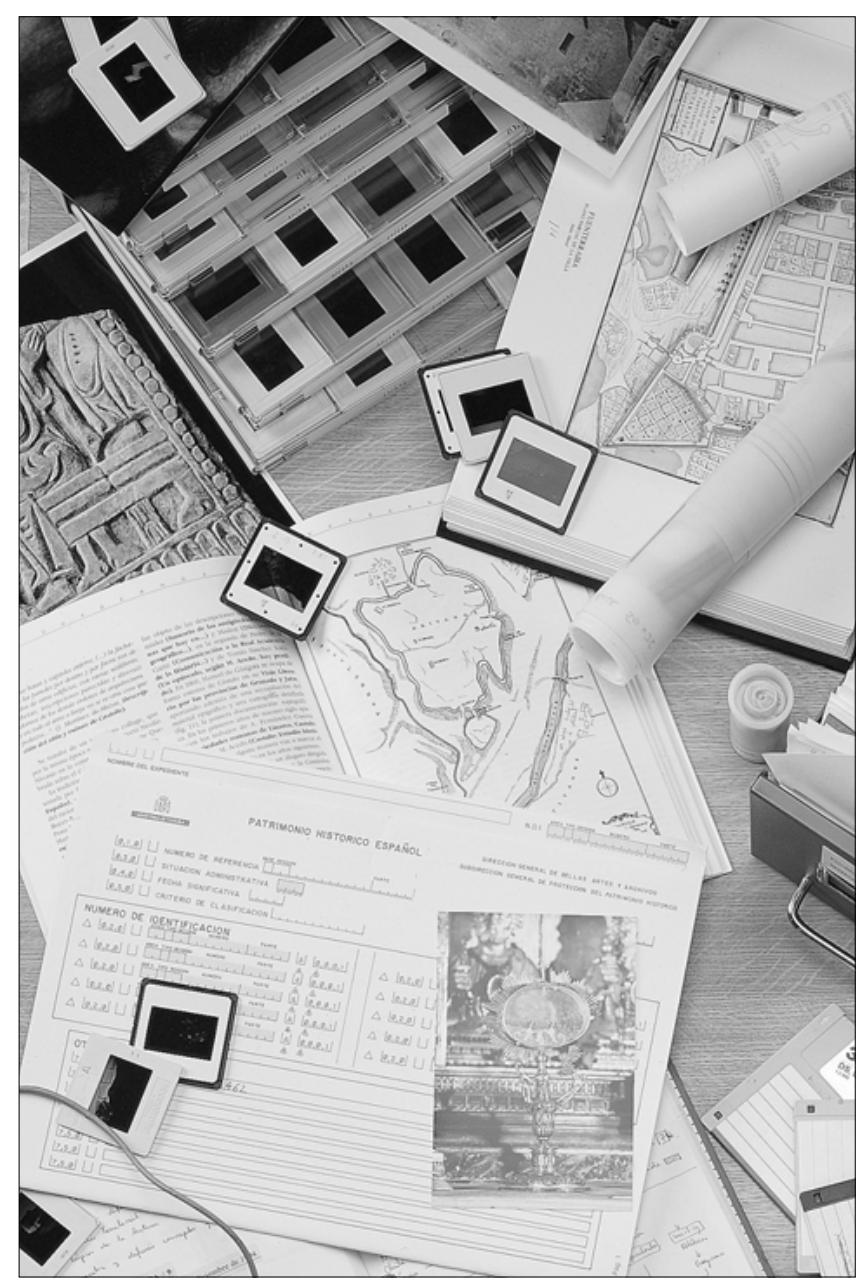

Es también necesario plantearse una revisión de la disciplina de la historia del arte con objeto de elaborar un método de trabajo que resulte adecuada para conseguir una correcta lectura científica del monumento, huyendo de los trabajos excesivamente amplios y poco prácticos para ser utilizados por un técnico que necesita respuestas concretas a preguntas concretas. Los estudios globales, las adscripciones a corrientes estilísticas o a períodos históricos muy precisos no pueden excluir lo fundamental a la hora de realizar un informe previo a la actuación en un Bien de Interés Cultural. El profundo análisis de la fábrica, el estudio directo del monumento, debe ser siempre prioritario ya que, como se ha expuesto anteriormente, se considera documento de civilización y cultura. El historiador del arte debe, pues, utilizar el monumento como una fuente documental básica para su estudio.

Esta reflexión no puede obviar el capítulo dedicado a las restauraciones anteriores. Las intervenciones posteriores a la configuración inicial del edificio forman parte inseparable de su historia y, por tanto, de su particular libro de fábrica. 


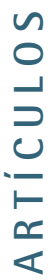

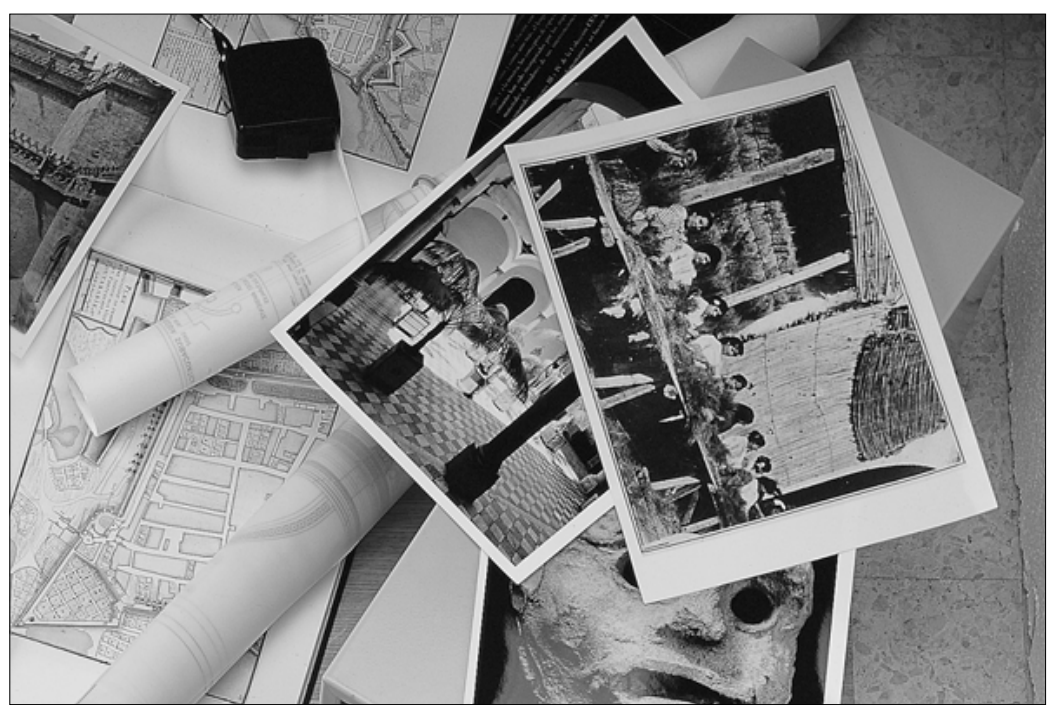

Se deben evitar prejuicios al respecto, y considerar que a pesar de la pretendida inocuidad de muchos procedimientos empleados en la labor restauradora, todas las actuaciones dejan una huella en la fábrica, incluso las más pretendidamente inocuas y reversibles. Por lo que este tema ha de ser afrontado sin hipocresías ni adscripciones a corrientes ni posturas, el no-método, la consciencia de que cada edificio es un individualidad que precisa de ser abordada sin ningún planteamiento hecho "a priori" para llegar a un profundo conocimiento científico del objeto sobre el que se ha de actuar es, posiblemente, la actitud más acertada:

“...habremos de ser todos conscientes de que cuando sea necesario adoptar nuevos elementos que ayuden a potenciar el uso y la significación del monumento, la mejor manera de garantizar que nuestra intervención sea histórica es que esté planteada y resuelta con los presupuestos culturales y con el lenguaje formal actual, es decir el lenguaje del momento histórico en el que se realiza" (I2).

Pues bien, en Galicia, salvo casos particulares, muy concretos y aislados, los proyectos de restauración no cuentan con una memora histórica del monumento. No se insiste de nuevo en la importancia que ésto tiene al tratarse de la actuación en un documento de civilización y cultura, puesto que se ha desarrollado ampliamente a lo largo de este trabajo, pero es interesante recordar que la eliminación, por falta de comprensión, de algún elemento de un edificio puede dejar "muda" para la historia una parte importante de la memoria colectiva que, plasmada en piedra, aporta no sólo datos históricoconstructivos, sino también referencias culturales y sentimentales del lugar en el que el monumento se emplaza.

En los proyectos a los que se acompaña una reseña histórica, la mayor parte de las veces, no está realizada por los técnicos adecuados; frecuentemente son los mismos arquitectos los que, de modo particular, inician una investigación para la que no están cualificados. Por otro lado, mientras que la documentación gráfica, en la mayoría de los proyectos consultados para este trabajo, es completa y contiene tanto los planos del estado inicial como las variaciones que se derivan de la actuación prevista, la documentación fotográfica, es prácticamente inexistente. Un reportaje fotográfico de la situación en la que se encontraba el edificio antes de la intervención, el avance de las obras y las soluciones proyectuales finales son documentos que no sólo están previstos en todas las normativas y recomendaciones internacionales sino que han de constituirse en fuentes documentales básicas, de vital importancia para poder afrontar -en el futuro- las contingencias que pueda sufrir el monumento.

\section{LA ACTUACIÓN ARQUITECTÓNICA}

El seguimiento de las obras y las transformaciones o alteraciones que se producen a lo largo de las mismas, en raras ocasiones están documentadas. Por ello, a menudo, cuando se realiza el trabajo de campo con objeto de realizar el estudio histórico-constructivo del edificio y comprobar los resultados de una restauración arquitectónica concreta, se ha podido constatar que pocas veces el proyecto coincide con la realidad. Se entiende que esta circunstancia no sólo es frecuente sino que, en cierto modo, es lógica. La práctica de la restauración monumental exige la dedicación, paciencia y pericia artesanal del equipo técnico que la lleva a cabo. Con la evolución de los distintos trabajos, los proyectos pueden ser modificados e incluso profundamente alterados ya que se considera francamente difícil prever totalmente cual es la situación real de la fábrica en una primera observación del edificio. 
Por todo ello, a menudo, los proyectos se convierten únicamente en un avance de intenciones que poco o nada tienen que ver con su resultado final. Resulta, no obstante, inadmisible que, si realmente todos los profesionales que actúan en el patrimonio cultural tienen consciencia de que el monumento es un documento, modificaciones no se reflejen en informes mensuales $y$, más tarde, en el informe final de las mismas. Si escasas son las memorias y los estudios previos al proyecto de restauración con documentación fotográfica del estado inicial, de la evolución de las obras y del estado final del monumento una vez que éstas han terminado, más raros son aún los informes finales de las obras.

Esta documentación es imprescindible, no sólo para que los historiadores del arte puedan seguir analizando y estudiando los monumentos en un futuro, sino para que otros arquitectos, en otras actuaciones, puedan resolver adecuadamente problemas planteados por soluciones que, consideradas como las más idóneas en el momento de la intervención, no han respondido adecuadamente con el paso de los años o que ellas mismas han provocado patologías imprevisibles en el edificio. Al no estar documentados los métodos utilizados pueden resultar incomprensibles cuando otros técnicos traten de abordar el problema.

\section{UNA NUEVA SITUACIÓN PARA UNA NUEVA DÉCADA}

Con las diferentes legislaciones autonómicas en materia de patrimonio se producen ciertos matices con respecto a este tema. Mientras que los estudios previos a la intervención arquitectónica y el informe final de obra son ignorados por la Ley 7/1990 del Patrimonio Histórico-Artístico del País Vasco, la Ley del 3 de julio de |99|, número |/9| del Parlamento de Andalucía, sobre el Patrimonio Histórico de Andalucía, publicado en el Boletín Oficial de la Junta de Andalucía número 59 al 13 de julio de 199|, en su artículo 22 se especifica lo siguiente:

I. "Los Proyectos de Conservación se ajustarán al contenido que reglamentariamente se determine, incluyendo, como mínimo la identificación del bien, la diagnosis de su estado, la propuesta de actuación, desde el punto de vista teórico, técnico y económico, y la descripción de la metodología a utilizar".

2. Los Proyectos de Conservación irán suscritos por técnico competente.

3. Corresponderá asimismo a técnico competente la dirección de las obras e intervenciones de conservación o restauración.

La Ley 9/1993, de 30 de septiembre, del Patrimonio Cultural Catalán, publicada en el B.O.E. número 264 del 4 de noviembre de 1993, de un paso adelante al citar en su artículo treinta y cuatro bajo el epígrafe Autorización de obras, apartado $3^{\circ}$ :

"Cualquier proyecto de intervención en un bien inmueble de interés nacional incluirá un informe sobre sus valores históricos, artísticos y arqueológicos y sobre su estado actual, y también de evaluación del impacto de la intervención que se propone".

Pero, es en la recientemente aprobada Ley del Patrimonio Cultural de Galicia, artículo treinta y nueve apartado tres, referido a los proyectos de intervención, cuando se hace expresa referencia a la necesidad de la participación de un profesional de la historia en los estudios previos a todo proyecto de restauración, y lo hace en los siguientes términos:

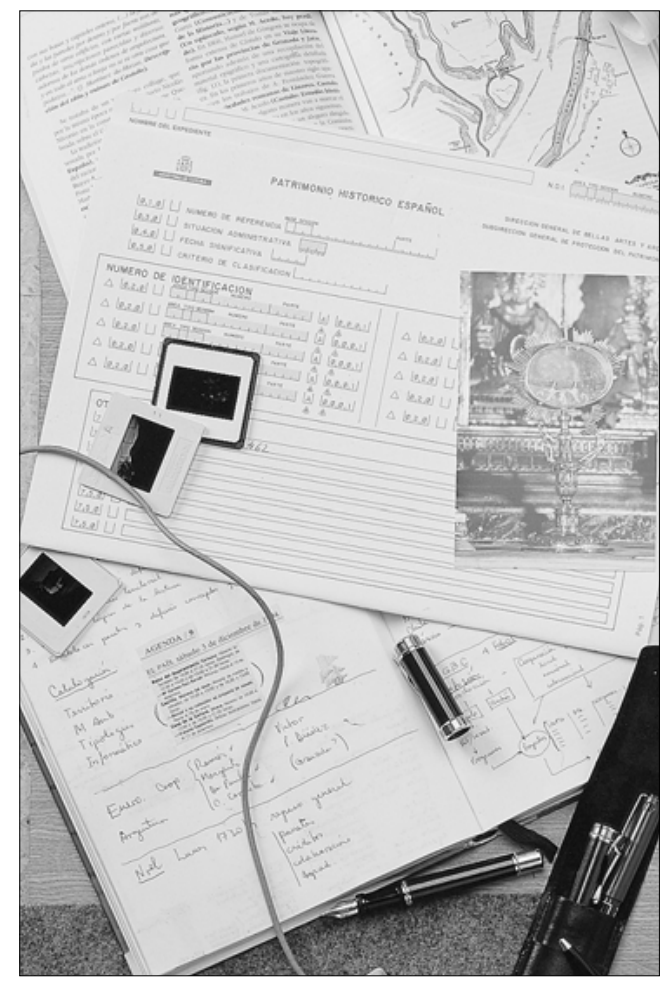

I. "Calquera proxecto de intervención nun ben inmoble declarado Ben de Interese Cultural deberá incorporar un informe sobre a súa importancia artística, histórica elou arqueolóxica, elaborado por técnico competente en cada unha das materias. Do mesmo xeito, deberase incluir en dito informe unha avaliación da intervención que se propón".

Pero especifica aún más:

"Una vez rematada a intervención, a dirección facultariva realizará unha pequena memorai na que figure, alomenos, descripción pormenorizada da obra executada e trataremos aplicados, así como documentación gráfica do proceso seguido" (13).

Esperamos que esta puntualización de la nueva Ley del Patrimonio Cultural de Galicia, contribuya a solucionar la situación actual de los profesionales de la 
historia ante las actuaciones en el patrimonio cultural de esta Comunidad Autónoma.

Es conveniente realizar un somero análisis de esta normativa ya que supone la materialización de un largo proceso que, iniciado en los años ochenta, se consolida al inicio de la nueva década para obtener su total maduración con la publicación de esta ley. Concede una especial importancia a la investigación de los elementos que constituyen el patrimonio cultural de esta Comunidad Autónoma, al establecer en el título preliminar, que el objeto de la presente ley es la protección, conservación, acrecentamiento, difusión y fomento del patrimonio cultural de Galicia, así como su investigación y transmisión a generaciones futuras.

Además, en el artículo sexto, referente a la protección exterior señala que la difusión exterior del patrimonio cultural de Galicia será impulsada por la Xunta de Galicia que fomentará los intercambios culturales que estime oportunos y promoverá, en el ámbito de sus competencias, el establecimiento de convenios y acuerdos con las demás comunidades autónomas, así como con los estados en los que se encuentren bienes integrantes del patrimonio cultural de Galicia.

En el título II instituye tres niveles diferentes de protección: los monumentos considerados Bienes de Interés Cultural, los Catalogados y los simplemente Inventariados. Además, los inmuebles se consideran integrados dentro de un contexto que es su territorio y no como elementos aislados, de igual forma, los proyectos de intervención se conformarán con informes pruridisciplinares dictados por profesionales de las distintas materias para garantizar con ello la conservación del bien, al tiempo que se dotan de un contenido claro y específico los planeamientos de los conjuntos históricos.

En el artículo once del capítulo primero, dedicado a los bienes de interés cultural, determina perfecta- mente lo que se debe considerar entorno de un monumento:

"No caso de inmobles, ademais, deberán figurar perfectamente definidas as súas relacións coa área territorial á que pertence, así como a protección dos accidentes xeográficos e elementos naturais que conformen o seu entorno, que aparecerá delimitado tamén graficamente, en atención á súa adecuada protección contemplación e estudio" (|4).

También, en el artículo cuarenta y cuatro define y especifica exhaustivamente, $y$ desde una perspectiva totalmente innovadora, el entorno de los Bienes de Interés Cultural, recogidos de un modo excesivamente genérico en el resto de las normativas, tanto nacional como autonómicas.

I. O contorno dos monumentos estará constituído polos inmobles e espacios lindantes inmediatos e, en casos excepcionais, polos non lindantes ou arredados, sempre que unha alteración deles poida afeta-los valores propios de ben de que se se trate, a súa contemplación, apreciación ou estudio.

2. O volumen, a tipoloxía, a morfoloxía e o cromantismo das intervencións no contorno dos monumentos no poden altera-lo carácter arquitectónico e paisaxístico da zona, nin perturba-la contemplación do ben.

3. Poderán expropiarse, e proceder ó seu derrumbamento, os inmobles que impidan ou perturben a contemplación dos monumentos ou dean lugar a riscos para estes.

Los avances en materia de restauración monumental, si bien ya se intuían a principios de los años ochenta, se van consolidando a medida que avanza la década para situarse, a principios de los años noventa, en una línea de trabajo cada vez más perfeccionada que culmina con la publicación de la Ley de Patrimonio Cultural de Galicia y que permite a los profesionales implicados en el tema afrontar el futuro con un cierto optimismo.

\section{Notas}

I. Viollet le Duc, E.: Dictionnaire raisonné de l'architecture française du XI au XVI siècle. B. Bauce-A. Morel, Paris I85468, t. VIII, "restauration", p. 33.

2. Ceschi, Carlo.: Teoria e storia del restauro. Mario Bulzoni Editori. Roma 1970, p. 211 .3. Brandi, Cesare. Teoría de la restauración. Alianza Editorial, S.A. Madrid 1989.

3. Brandi, Cesare. Teoría de la restauración. Alianza Editorial, S.A. Madrid 1989.

4. Carta de Venecia, mayo 1964:: Preámbulo.

5. Carta de Venecia, mayo 1964.: artículo I.

6. Idem, artículo 9

7. Jiménez Martín, A.: Carta del Restauro'72. C.O.A.S. Sevilla 1982, p. 81.

8. Déclaration d'Amsterdam, octubre 1975.: Préface. Consideration i.

9. Ley 16/1985, de 25 de junio, del Patrimonio Histórico Español.: Artículo treinta y nueve.
10. González Moreno-Navarro, A.: “La investigación histórica en el proceso de intervención en los monumentos" en Historia y Arquitectura. La recerca històcia en el procés d'intervenció en els monumentes. Servei de Catalogació y Conservació de Monuments. Barcelona 1986, p. 155.

I I. Azcárate, A./Fernández de Jáuregui, A./Nuñez, M.: "Documentación y análisis arquitectónico en el País Vasco. Algunas experiencias llevadas a cabo en Álava. España" en Informes de la Construcción, $n^{\circ} 5$. Leer el documento construido. Consejo Superior de Investigaciones Científicas, Madrid 1995, p. 74.

12. González Moreno-Navarro, A.: La investigación histórica..., op. cit., p. 155.

13. Ley del Patrimonio Cultural de Galicia.: Artigo 39, apartado 2. D.O.G. N²14, 8 de noviembre de 1995.

14. Ley del Patrimonio Cultural de Galicia.: op. cit., artigo II. Contido de expediente de declaración, apartado b. 